\title{
Barriers and facilitators to the use of digital systems in primary care to deliver physical activity advice using COM-B and theoretical domains framework
}

Paulina Bondaronek ${ }^{12^{2}}$, Samuel J. Dicken ${ }^{12}$, Seth Singh Jennings ${ }^{2}$, Verity Mallion², Chryssa Stefanidou'1.2

- Office for Health Improvement and Disparities, London, SW1H OEU

${ }_{2}^{2}$ Public Health England, Wellington House, London, SE1 8UG (until September 2021)

* Author correspondence: Paulina Bondaronek: Bondaronek,

Paulina.Bondaronek@dhsc.gov.uk

\section{Abstract: 495 words}

Manuscript 6220 words (excluding abstract, captions and references)

Tables: 2 (3 including supplementary materials) 


\begin{abstract}
Background: Physical inactivity is a leading risk factor for many health conditions, including cardiovascular disease, diabetes and cancer; increasing physical activity (PA) is therefore a public health priority. Healthcare professionals (HCPs) in primary care have been identified as being pivotal in addressing physical inactivity, yet few HCPs provide PA advice to patients. There can be obstacles to delivering PA advice, including a lack of time, confidence or knowledge. Digital technology has the potential to overcome obstacles and facilitate delivering PA advice. However, it is unknown if and how digital systems are used to deliver physical activity advice in primary care consultations, and what factors influence their use.
\end{abstract}

The aim of this study was to understand the use of digital systems to support primary care consultations and to identify the barriers and facilitators to using these systems.

Methods: 25 semi-structured interviews were conducted with HCPs in primary care. Professionals were sampled purposively based on profession (general practitioners, practice nurses and healthcare assistants), prevalence of long-term conditions within their practice area, and rural-urban classification. Data were analysed using thematic analysis to identify influences on the use of digital systems. Themes were categorised using COM-B and the theoretical domains framework (TDF) to identify the barriers and facilitators to using digital systems to support the delivery of PA advice in primary care consultations.

Results: Identified themes fell within eight TDF domains (linked COM-B component follows in parentheses): Knowledge (Psychological Capability), Skills (Psychological Capability), Environmental Context and Resources (Physical Opportunity), Social Influence (Social Opportunity), Beliefs about Capabilities (Reflective Motivation), Beliefs about Consequences (Reflective Motivation), Reinforcement (Automatic Motivation), and Emotions (Automatic Motivation). The most prominent barrier/facilitator within psychological capability was 'having the skills to use digital systems'. 'Training in the use of digital systems' was also mentioned several times. The most notable barriers/facilitators within physical opportunity were 'time constraints', the 'efficiency of digital systems', the 'simplicity and ease of use' of digital systems, and 'integration with existing systems'. Other physical opportunity barriers were 'lack of access to digital systems' and 'technical support in the use of digital systems'. With respect to social opportunity, a notable barrier was the sense that 'digital systems reduce interpersonal communications' with patients. 'Patient preference' was also mentioned as a barrier/facilitator. Several important barriers and/or facilitators were within reflective motivation, including 'confidence to use digital systems', 'beliefs about the usefulness of digital systems', the 'belief that digital systems 'are the way forward", 'beliefs related to data privacy and security concerns', and 'perceptions about patient capabilities'. With respect to automatic motivation, barriers/facilitators included 'familiarity and availability' regarding digital systems, and the fact that digital systems 'prompt behaviour'.

Conclusions: A variety of influences were identified on the use of digital systems to support primary care consultations. These findings provide a foundation to design a digital system that addresses the barriers and leverages the facilitators to support PA advice provision within primary care, to elicit patient behaviour change and increase PA.

Key words: physical activity; COM-B, TDF, primary care professionals; healthcare professionals; physical activity advice 


\section{Introduction}

Physical inactivity is a leading cause of death and non-communicable disease worldwide (World Health Organisation, 2009). Being physically active can reduce the risk of all-cause mortality and help to prevent and manage a wide range of long-term conditions, including cardiovascular disease (CVD) and some cancers and neurodegenerative diseases (Department of Health and Human Services, 2018; GOV.UK, 2019). Moving from a state of inactivity to meeting UK government physical activity (PA) recommendations of 150 minutes per week of moderate to vigorous PA can reduce the risk of CVD incidence by $17 \%$, CVD mortality by $23 \%$ and type 2 diabetes incidence by $26 \%$, even after adjusting for bodyweight (Wahid et al., 2016). Furthermore, PA has positive impacts on mental health and well-being (Department of Health and Human Services, 2018; GOV.UK, 2019).

Identifying effective methods to increase PA in the population is therefore of great importance. One approach is to deliver PA advice to patients within primary care. As a trusted source of health-related information that frequently interacts with large proportions of the population, healthcare professionals (HCP) within primary care have pivotal roles in encouraging greater PA (Jones et al, 2019, Hall et al, 2021). As many as one in four people say they would be more active if they were advised by a GP or a nurse (Orrow et al, 2012).

Delivering brief PA advice in primary care have been shown to be cost effective (Vijay et al, 2016; Milton et al, 2021), with positive impacts on PA and health outcomes (Vuori et al, 2013; Goryakin et al, 2018). As such, the National Institute for Health and Care Excellence recommends that brief PA advice is given in primary care (NICE, 2013). However, the delivery of brief PA advice in primary care is not routine and remains to be fully established. Only one third of all patients report receiving such advice (Loprinzi et al, 2014, Jones et al, 2019). Despite knowledge amongst HCPs that increasing PA is beneficial for their patients (Wattanapisit et al, 2019), a number of reviews and studies have identified key obstacles that limit the delivery of PA advice in primary care. Important obstacles include a lack of knowledge (of national PA guidelines, of how to deliver advice, of what advice to give, of how to communicate effectively) (Chatterjee et al, 2017; Goryakin et al, 2018; Wattanapisit et al, 2019; Huntington et al, 2020, Hall et al, 2021), a lack of tools or resources (Hall et al, 2021), an inability to follow up on patients (Wattanapisit et al, 2019; Hall et al, 2021), the perceived readiness and motivation of the patient to change (Albert et al, 2020; Hall et al, 2021), a lack of confidence (Belanger et al, 2017) and time constraints (Gustavsson et al, 2018; Wattanapisit et al, 2019; Hall et al, 2021).

Establishing the routine delivery of PA advice in primary care requires overcoming such obstacles. A promising new avenue is the use of digital systems, which may provide opportunities to facilitate the delivery of PA advice in primary care. Digital systems can include electronic (eHealth) and mobile (mHealth) interventions, and online training (elearning). The World Health Organisation (WHO) has highlighted the importance of utilising innovative digital technologies to promote PA and reduce sedentary behaviour in its Global Action Plan on Physical Activity (World Health Organisation, 2018).

Digital systems have previously been used to deliver PA advice in primary care (Wattanapisit et al, 2020a; Wattanapisit et al 2020b; Brannan et al, 2019), either by supporting (Agarwal et al, 2020) or replacing (Degroote et al, 2020) face-to-face delivery of PA advice. Digital systems appear to have potential utility for increasing PA by supporting the delivery of PA advice (van der Weegen et al, 2015; Boudreau et al, 2020; Wattanapisit et al, 2020a). However, primary care HCPs have mixed views on the usability of digital systems, with barriers to their use including technical issues and complexity, disruption to service workflow and increasing workload (Verwey et al, 2015; Granja et al, 2018; Neudorf et al, 2020; Wattanapisit et al, 2020). 
In order to determine the value of digital systems to support the delivery of PA advice and how to optimise their development and integration, it is important to fully understand the existing challenges with delivering PA advice, the influences on using digital systems, the required characteristics of a digital system and the opportunities to incorporate digital systems into existing practice. However, there is a paucity of evidence surrounding the obstacles that face using digital systems to deliver PA advice. Studies rarely use a behavioural framework to systematically identify barriers and facilitators, or instead focus on patient perspectives (Wattanapisit et al, 2020a). Studies have only considered specific digital systems such as eHealth or mHealth interventions, and not all potential digital systems.

Systematic approaches to investigating the factors that influence health-related behaviour and professional practice can be facilitated through the use of behavioural science tools. The capability, opportunity, motivation - behaviour (COM-B) system is a model of behaviour change that helps to understand the influences of a behaviour (Michie et al, 2011). A related model is the Theoretical Domains Framework (TDF), which can be mapped onto COM-B to further categorise influences into the facilitators that increase, and the barriers that hinder, the behaviour (Atkins et al, 2017). COM-B and TDF have been used widely in previous studies to synthesise findings on the barriers and facilitators for a range of behaviours, including a review of physician-reported barriers to using evidence-based recommendations for low back pain (Hall et al, 2019), a review of the factors influencing implementation of screening and brief interventions for alcohol in primary care (Rosario et al, 2021), and specifically for promoting PA by HCPs (Albert et al, 2020). In this study, we used COM-B and TDF to systematically map the barriers and facilitators to using digital systems to deliver PA advice in primary care.

\section{Aims and objectives}

The present study aims to understand the use of digital systems to support primary care consultations and to identify the barriers and facilitators to the use of these systems to deliver PA advice.

The specific objectives were:

(1) to gain insights on the use of digital systems within primary care settings to understand the influences on their use to deliver PA advice.

(2) to systematically map the influences using COM-B and TDF to understand the barriers and facilitators to using digital systems within primary care to deliver PA advice. 


\section{Methods}

\section{Study Design}

This was an exploratory qualitative study drawing on interviews with HCPs in primary care.

\section{Sample}

A sample of HCPs was recruited purposively (by a third-party recruiter) based on profession (GPs, practice nurses and healthcare assistants (HCAs)); prevalence of long-term conditions within the area (in particular, obesity, depression, hypertension, coronary heart disease and diabetes), and rural-urban classification. During recruitment, HCPs were also screened to ensure a range of experience levels (based on length of time working within primary care, selfreported levels of delivering PA advice and self-reported digital skills). Data collection ceased once saturation of themes was reached, which resulted in a total of 25 interviews being completed, transcribed and coded.

\section{Inclusion criteria}

To be included, study participants had to be a GP, nurse or HCA; must have worked in general practice; must have worked in the UK healthcare system for a minimum of one year; must be an English speaker; and must be 18 or older.

\section{Data collection}

Semi-structured interviews lasting 60-90 minutes were conducted via telephone in March and April 2020. This timeframe coincided with the introduction of the first COVID-19 protocols in the UK, including the national lockdown on March 23, 2020, hence all study interviews were conducted via telephone. In line with ethical guidelines, written informed consent was obtained from participants prior to commencing the interview. A topic guide based on COM-B (Michie et al, 2014) was used by interviewers to support discussions.

During interviews, HCPs were asked a series of open-ended questions about their routines and working days; the systems and resources they use routinely to identify patients and to deliver and record advice; their capability, opportunity and motivation to use these systems and resources effectively; any barriers to using these tools and resources and suggested solutions and improvements to overcome them.

The topic guide included various prompts and follow-up questions to help elicit data relevant to the research questions.

\section{Data management and analysis}

The interviews with the $25 \mathrm{HCPs}$ were recorded on password protected and encrypted machines to ensure data privacy and security. Recordings were uploaded to the encrypted, password-protected Citrix platform to be transcribed verbatim by a third-party provider, and the original recordings were then deleted from the study team's systems. The third-party provider removed any identifying information during transcription and returned anonymised transcripts to the study team for data analysis.

Anonymised transcripts were then imported into Excel for analysis. Participant responses were broken down into constituent parts to analyse distinct thoughts and ideas independently. Content analysis informed by the COM-B model was used to analyse the data. One researcher read through each of the 25 transcripts, extracted data relevant to the use of digital systems, 
and inputted the data into an Excel spreadsheet. We cast a wide net in our understanding of 'digital systems' and included references to the use of any computer- or mobile technologyaided system. In total, 165 comments from participants relating to the use of digital systems were recorded. Another member of the research team inductively coded the data, line by line, using constant comparison techniques within and between codes to ensure that they accurately reflected the material. Codes were then examined for similarities and grouped, inductively, into themes regarding barriers and facilitators for the use of digital systems to identify patients and to deliver and record PA advice. The themes that emerged from this process - i.e. the ones that were identified as being important - were either articulated by multiple respondents (high frequency) or were articulated particularly clearly and forcefully (elaboration); or both. Once the data was coded to be either a barrier, facilitator or both, data was deductively classified under the COM-B model (Michie et al, 2011) and TDF (Cane et al, 2012) to systematically understand these behaviours and needs. When multiple COM-B components and themes could be used to code data, further data segmentation was considered if it was deemed that the existing data segment contained discrete thoughts. Further data segmentation was reported during coding through putting a forward slash (/) inbetween COM-B components and themes.

Classifying data into COM-B components followed expert guidelines (Michie et al, 2014): one researcher was tasked with classifying all of the extracts, and a second researcher coded $20 \%$ of extracts to highlight and resolve any discrepancies in the coding. A random number generator was used to provide a random sequence of Excel cell numbers containing data segments that would be coded by the second researcher. After independently completing one round of coding, the two researchers met via video call to discuss codes. Any disagreements over codes were discussed until consensus was reached and the dataset was updated accordingly. Similarly, the decision to split data segments was discussed between researchers until agreement was reached. 


\section{Results}

Participant characteristics are presented in table 1. Participants tended to be practice nurses or HCAs, older and working in an urban setting, with a range of primary care experience.

Table 1: Summary of participant characteristics

\begin{tabular}{|c|c|}
\hline $\mathbf{N}$ & 25 \\
\hline Profession & \\
\hline GP & $6(24 \%)$ \\
\hline Practice Nurse & $10(40 \%)$ \\
\hline $\mathrm{HCA}$ & $9(36 \%)$ \\
\hline Age $(\mathrm{N}=23)$ & \\
\hline $18-30$ & $3(13 \%)$ \\
\hline $31-50$ & $7(30 \%)$ \\
\hline $50+$ & $13(57 \%)$ \\
\hline Primary care experience & \\
\hline $1-10$ years & $7(28 \%)$ \\
\hline $11-20$ years & $9(36 \%)$ \\
\hline $20+$ years & $9(36 \%)$ \\
\hline Location $(\mathrm{N}=24)$ & \\
\hline Rural & $7(29 \%)$ \\
\hline Suburban & $1(4 \%)$ \\
\hline Urban & $16(67 \%)$ \\
\hline
\end{tabular}

23 respondents for age, 24 respondents for location.

The barriers and facilitators on the use of digital systems to deliver PA advice in primary care are presented in table 2. Additional participant responses are provided in supplementary table 
S1. Important themes were identified within psychological capability, physical and social opportunity and reflective and automatic motivation.

Table 2: Important themes identified by participants

\begin{tabular}{|c|c|c|c|}
\hline \multicolumn{2}{|l|}{ COM-B } & TDF & Themes \\
\hline \multirow[t]{3}{*}{ Capability } & \multirow[t]{2}{*}{ Psychological } & \multirow[t]{2}{*}{$\begin{array}{l}\text { Knowledge } \\
\text { Skills }\end{array}$} & $\begin{array}{l}\text { Having the skills to use } \\
\text { digital systems }\end{array}$ \\
\hline & & & $\begin{array}{l}\text { Training in the use of digital } \\
\text { systems }\end{array}$ \\
\hline & Physical Capability & Physical skills & $\begin{array}{l}\text { Not reported as a significant } \\
\text { influence }\end{array}$ \\
\hline \multirow[t]{8}{*}{ Opportunity } & \multirow[t]{6}{*}{ Physical } & \multirow{6}{*}{$\begin{array}{l}\text { Environmental } \\
\text { context and } \\
\text { resources }\end{array}$} & Efficiency of digital systems \\
\hline & & & $\begin{array}{l}\text { Integration with existing } \\
\text { systems }\end{array}$ \\
\hline & & & $\begin{array}{l}\text { Lack of access to digital } \\
\text { systems }\end{array}$ \\
\hline & & & Simplicity and ease of use \\
\hline & & & $\begin{array}{l}\text { Technical support in the use } \\
\text { of digital systems }\end{array}$ \\
\hline & & & Time constraints \\
\hline & \multirow[t]{2}{*}{ Social } & \multirow[t]{2}{*}{ Social Influences } & $\begin{array}{l}\text { Digital systems reduce } \\
\text { interpersonal } \\
\text { communication }\end{array}$ \\
\hline & & & Patient preferences \\
\hline \multirow[t]{7}{*}{ Motivation } & \multirow[t]{5}{*}{ Reflective } & \multirow[t]{3}{*}{$\begin{array}{l}\text { Beliefs about } \\
\text { capabilities }\end{array}$} & $\begin{array}{l}\text { Beliefs related to data } \\
\text { privacy and security } \\
\text { concerns }\end{array}$ \\
\hline & & & $\begin{array}{l}\text { Confidence to use digital } \\
\text { systems }\end{array}$ \\
\hline & & & $\begin{array}{l}\text { Perceptions about patient } \\
\text { capabilities }\end{array}$ \\
\hline & & \multirow[t]{2}{*}{$\begin{array}{l}\text { Beliefs about } \\
\text { consequences }\end{array}$} & $\begin{array}{l}\text { Beliefs about the usefulness } \\
\text { of digital systems }\end{array}$ \\
\hline & & & $\begin{array}{l}\text { Belief that digital systems are } \\
\text { 'the way forward' }\end{array}$ \\
\hline & \multirow[t]{2}{*}{ Automatic } & \multirow{2}{*}{$\begin{array}{l}\text { Reinforcement } \\
\text { Emotions }\end{array}$} & Familiarity and availability \\
\hline & & & Prompt behaviour \\
\hline
\end{tabular}

Psychological Capability

Having the skills to use digital systems was reported by numerous respondents as an important factor influencing the use of digital systems in primary care to deliver PA advice, being described as both a barrier and a facilitator. While some respondents reported feeling confident in their digital skills and ability to use digital systems, a number of HCPs discussed how not having the skills and/or the confidence to use digital systems may act as a barrier to their use. Although providing appropriate training may act as a facilitator for the use of digital systems within primary care, HCPs overwhelmingly discussed a lack of adequate training to provide them with the skills and confidence to use digital systems (barrier). Hence, training in 
the use of digital systems was identified as another notable theme. Due to the lack of formal training, some staff discussed having to rely on other members of staff within the practice with more experience using digital systems to teach them how to use the systems. HCPs may therefore benefit from receiving some form of training on how to use digital systems.

"I'm pretty good but EMIS is one of those things that there is always something more to learn really. You can learn the basics in quite a short period of time but I am still finding things that I think, God, if l'd have known that a few years ago, that would have saved me an awful lot of time."

(Nurse, 31-50 years)

"There is no formal training by and large, other than you may get sent a document of how to do something. So we have relied upon one of our staff members who, for want of a better word, is like an IT manager who will take overall charge of these things and oversee their introduction and development, and disseminate that information as a practice and ensure that we're all up to speed. So you need to have one person who has that as their responsibility and role within the practice."

(GP, 50+ years)

\section{Physical opportunity}

Physical opportunity was the most frequently coded COM-B component, reflecting the fact that it was associated with the greatest number of themes emerging from the data (table 1). A barrier to using digital systems that was mentioned a number of times was the issue of time constraints. It was clear that HCPs often experienced time pressures and did not always have the time to consider other ways of working (i.e. they will often default to what they are used to). On the flip side, there was a feeling that digital systems can save time by increasing efficiency, hence the theme of efficiency of digital systems. Digital systems were reported to make more efficient use of the limited time available for consultations, allowing data to be more easily captured and stored in comparison to manual data recording (facilitator).

"When you're so busy and flat out, you don't have sometimes that time to just sit back and reflect and think, well, is there another way I could be doing this more efficiently?"

(GP, 50+ years)

"You have your clinics. You have your QOFs to do. You want to follow the NICE guidelines on every patient with a long-term condition. We have all of those responsibilities as well as the urgent on the day requests. Jiggling time is always a factor."

(Nurse, 50+ years)

Relating to the themes of efficiency of digital systems and time constraints, was the theme of simplicity and ease of use, which may act as either a barrier or facilitator, depending on the design and subsequent functionality of the digital system. For example, templates need to be as simple as possible, quick to use, easy to navigate, and must also facilitate the collection of all mandated/important data. Another way in which digital systems can be made easier to use is if they allow for integration with existing systems. This was also highlighted by respondents as both a barrier and facilitator. This was categorised as a separate theme as it was deemed to be a specific requirement. Another physical opportunity barrier to the use of digital systems was the lack of access to digital systems in some areas. A final physical opportunity consideration was the presence of technical support in the use of digital systems; respondents reported that having technical support to hand facilitated the use of digital systems. 
"It's much easier. Much easier than sitting there writing things out. You can click. It gives you more time to do other things. It gives you more time with the patient. You're not spending lots of time writing things out. You are more for the patient than you are writing things down."

(HCA, 31-50)

"I think sometimes in general practice the issue is we don't have much time... So I think any way in which we can reduce the number of clicks, to put it simply, the better, and if this system was generated automatically, it flags it up, then that would be better than having to deal with all those issues and then think about doing something else on top as well. I think the easier to use, the quicker to use, the less steps involved the better really."

(GP, 31-50 years)

"... it would have to be something that would be compatible with the system that we're using, and unfortunately l'm trying to get an ECG machine to be compatible with EMIS. So it's all about compatibility and whether one talks to the other."

(HCA, 50+ years)

\section{Social opportunity}

Barriers and facilitators relating to social opportunity were less frequently discussed by HCPs. However, there was an indication that some HCPs felt that digital systems reduce interpersonal communications with patients (barrier). One respondent also said that their propensity to use digital systems may be influenced by patient preferences (barrier or facilitator).

"Part of me doesn't mind but other times I think, Oh gosh I feel I'm looking at a computer screen rather than looking at a patient. I wasn't trained to do that; I'm very old school as well because I trained back in the eighties so I don't mind using it, I appreciate we have to move on with the times but I don't like it too much because I find that I'm watching the screen and making sure I've got everything that I need to fill on there without actually looking at the patient and just talking to them properly."

(Nurse, $50+$ years)

\section{Reflective Motivation}

One of the clearest themes under reflective motivation to emerge from the data was that of confidence to use digital systems. All respondents who mentioned confidence discussed it as a facilitator; expressing that they had the confidence required to use such systems. However, it is implicit in the notion of confidence that one could just as easily have it, as not have it; a lack of confidence would of course be a barrier to the use of digital systems. It should be highlighted that confidence to use digital systems often goes hand-in-hand with having the digital skills to use digital systems, as having the skills to do something tends to breed confidence, while not being confident may be an indication of a lack of skills. Despite this overlap, skills and confidence are different influences, and are coded differently within COM$\mathrm{B}$, which explains why some quotes in supplementary table S1 could seemingly fit into either.

\section{"I mean I'm of the generation which is fairly IT savvy, so I feel quite confident."}

(GP, 31-50 years)

Another theme that could be both a barrier and a facilitator for the use of digital systems was beliefs about the usefulness of digital systems. If someone believes that a system has utility, they will be more inclined to use it (facilitator), whereas if they believe the system is not useful or indeed a hindrance, they may be disinclined to use it (barrier). The majority of respondents who mentioned the usefulness of the digital systems within their practice felt that such systems 
were useful, but this was not unanimous. A common belief discussed by HCPs with positive views towards the usefulness of digital systems was that these systems save time and increase efficiency by, among other things, facilitating the sharing of data with secondary healthcare providers. It was also mentioned that digital systems also present unique opportunities to provide varied care to patients digitally when there is limited opportunity to provide physical care to patients face-to-face. However, others said that templates do not provide useful options and may be too time consuming to use within consultations, and that patients might also benefit from having a physical copy of advice.

'We can even do things like video consultations now which I think we've had to embrace because of the current situation with COVID. I think it will change the way we practise ongoing because we can see the efficiencies of these. I think the model of general practice personally is going to change hugely after this because we can see we can do things safely and differently and more efficiently."

(GP, 50+ years)

"I don't find the template is particularly useful... I don't think it's very useful in the information that it's asking for. Then the options it gives you, do you want to refer them to the health trainer? Nearly everybody will say no to that because it's too involved. It's too time consuming."

(HCA, $50+$ years)

A related theme to beliefs about the usefulness of digital systems was the belief that digital systems 'are the way forward'. Sometimes this theme overlapped with beliefs about the usefulness of digital systems; respondents said that digital systems were the way forward and then went on to support this with reasons based on usefulness, but sometimes it appeared to be offered as a reason in its own right. The belief that digital systems 'are the way forward' was a facilitator to the use of digital systems.

"The opportunities are there aren't they, we're moving forward and everything's IT and it's the way forward, for patients as well, apps and doing everything online and using phones,"

(Nurse, $50+$ years)

Beliefs related to data privacy and security concerns was another theme under reflective motivation that emerged from the data. This theme was both a barrier and facilitator to the use of digital systems, depending on the particular belief of each respondent. Some felt that digital systems improved security around patient data by reducing mistakes, while others said that safety features required to ensure patient safety within digital systems could act as a barrier to their use.

“They're just safer, and they protect patient confidentiality, and they're safer to use, things we can audit trails, process it all, and obviously check if anything goes wrong, if there was a fax it may reject or get sent somewhere else if the number was wrong."

(HCA, 18-30 years)

A final theme under reflective motivation were HCPs' perceptions about patient capabilities, and again this was both a barrier and a facilitator. A number of respondents suggested that their inclination to use digital systems during a consultation would depend on the technical capabilities of the patient in question.

"My dad, he needs everything explained manually and wouldn't go near a computer for him, I'd need to spend more time with him, to discuss a questionnaire l'd need to print it out and go through it with him, even phones."

(Nurse, 50+ years) 


\section{Automatic Motivation}

A common facilitator to the use of digital systems within primary care relating to automatic motivation was that digital systems, specifically templates, prompt behaviour. For instance, templates were described as useful as they provide guidelines and tick boxes to prompt HCPs to ask relevant questions and ensure nothing is missed during consultations. Another theme under automatic motivation, which was mentioned by a few respondents, was familiarity and availability. One respondent said that they used specific digital systems because it was what was available to them and was what they had always used, while another said that newly introduced digital systems are a bit daunting, but that the feeling subsides over time as they become familiar.

"They're optional, yes... I choose to use them, yes... It's easier and I feel like it's more thorough, and when it's a busy day especially, it's nice to just have that as a prompt."

(HCA, 18-30 years)

"I mean, sometimes when you first learn them, it is a bit daunting. You think, 'oh', and you're looking through them, but once you've done it a few times, you get a rhythm... As I said, if you go through every box, you can't go wrong."

(HCA, 31-50 years) 


\section{Discussion}

In this study, we identified important themes on the use of digital systems to support the delivery of PA advice within primary care, based on high frequency or elaboration, or both. Barriers and facilitators included skills and training to use digital systems, efficiency of digital systems including their integration and ease of use, patient preferences, confidence to use digital systems, beliefs about usefulness of digital systems, perceptions about patient capabilities and data privacy and security concerns. Time and reducing interpersonal communication were barriers, and the use of digital systems to prompt behaviour, the belief that digital systems are the way forward and having technical support were facilitators, to using digital systems to deliver PA advice.

This qualitative study expands upon previous findings on the barriers and facilitators to delivering PA advice in primary care (Albert et al, 2020; Hall et al, 2021), by using a behavioural framework to systematically identify the barriers and facilitators to using digital systems to deliver PA advice in primary care. The findings from this study indicate that important influences relating to knowledge, time and confidence for delivering PA advice are also important for using digital systems. But, other themes including the efficiency and integration of digital systems and data privacy and security concerns are important influences specifically for using digital systems in this context. As for delivering PA advice, there was variability across HCPs as to whether themes were barriers, facilitators, or both, to using digital systems.

The mixed views regarding the usability and utility of digital systems emerging from this study builds upon previous findings for eHealth interventions to deliver PA advice. Similarly, some HCPs find eHealth interventions useful and easy to use, but others perceive eHealth interventions to be time consuming or ineffective, with technical issues, inexperienced staff and the complexity of programmes being barriers to their use (Neudorf et al, 2020; Wattanapisit et al, 2020a; Wattanapisit et al, 2020b; Hall et al, 2021).

As with delivering PA advice (Lamming et al, 2017; Hall et al, 2021), time was a barrier to using digital systems, which is closely related to its efficiency and ease of use. Cumbersome digital tools that are poorly integrated slow down work and disrupt workflow, creating a barrier to their use to support delivering PA advice (Granja et al, 2018; Poppe et al, 2018; Wattanapisit et al, 2021a). A digital tool needs to be simple, easy and time-efficient to fit within short consultations (Poppe et al, 2018; Wattanapisit et al, 2021a), but also versatile, given that timeconstraints are likely to vary depending on consultation length. In agreement with the results of this study, integrating an eHealth tool into existing medical programmes and workflow is important to facilitate its use (Nápoles et al, 2016; Poppe et al, 2018; Wattanapisit et al, 2021a). The ability of a digital system to facilitate (or hinder) delivering PA advice therefore depends on how simple, effective, and well-integrated the system is.

Many participants in this study agreed that digitisation was the way forward, providing an efficient, simple and easy-to-use solution. However, interviews were conducted during the COVID-19 pandemic, where face-to-face consultations had been cancelled. Prior to COVID19 , the use of digital systems in place of face-to-face PA advice was previously identified as a barrier in terms of interpersonal communication, with HCPs preferring face-to-face communication (Granja et al, 2018). In this study, some participants highlighted that video consultations facilitated giving PA advice to those unable to attend in person. But on the other hand, participants indicated that in-person digital systems could be a barrier if the system excessively detracted from interacting with the patient. Digital systems can facilitate PA advice when they improve communication, which could be through providing templates with recommendations, set phrases or prompts. Digital systems could be used to generate personalised recommendations in an appropriate language to facilitate delivering PA advice 
(Wattanapisit et al, 2021a), which can be time-efficient by utilising tools prior to the consultation ((Christian et al, 2011; Mendes et al, 2020; Wattanapisit et al, 2021a; Poppe et al, 2018; O'Regan et al, 2021). MHealth tools can perform important tasks such as diagnosis, helping to reduce workload (Wattanapisit et al, 2020b). For example, digital tablets in the waiting room can save time by automating collection of routine data and performing health screening (Nápoles et al, 2016), which has the flexibility to be able to accommodate discussions across varying durations of consultations (Wattanapisit et al, 2020a) and support discussions with patients with limited health literacy (Wattanapisit et al, 2021c).

Previous studies show that knowledge, training or access to educational resources are common barriers and facilitators to delivering PA advice (Goryakin et al, 2018; Law et al, 2020; Hall et al, 2021). This study builds upon these findings by indicating that knowledge and training are also barriers and facilitators to using the digital system itself. Participants pointed out a current lack of technical support for using digital systems. There can be educational barriers and technical difficulties to using digital tools such as tablets or apps (Poppe et al, 2018). Delivering PA advice increases the workload of HCPs (Albert et al, 2020), and digital systems have the potential to facilitate delivering PA advice through improvements in efficiency and ease of use. But, the benefit of a digital system in reducing workload is likely dependent on the quality of training given to use digital systems, and the resources within it. Digital elearning systems are already used to provide PA education (Netherway et al, 2021), and training to increase knowledge, confidence and skills to deliver PA advice, such as the Moving Healthcare Professionals Programme (Brannan et al, 2019). A systematic review indicated that mobile tools may facilitate PA promotion by addressing knowledge and resource barriers (Albert et al, 2020), by providing a centralised, integrated tool for easy access to PA resources (Vishnubala et al, 2021). However, these systems do not provide training in the digital system itself. Provision of education and training in digital systems should also be considered if they are being used to support delivering PA advice.

Previous studies identify patient-related factors as an important theme affecting the motivation of HCPs to give PA advice, with patient abilities to use digital systems, preferences and readiness to change being barriers and facilitators (Wattanapisit et al, 2021a). In this study, patient motivation to change was not an important theme to using digital systems, but HCPs perceptions of patient motivation to use a digital system was a barrier or facilitator to their use, depending on whether patients preferred physical prints or had the technical capability to use a digital system. A lack of print materials in itself has also been cited as a barrier to delivering brief PA interventions (Campbell et al, 2012; Lamming et al, 2017). Importantly, HCPs use their subjective perception of patient capability and motivation to change to determine whether to deliver PA advice (Hall et al, 2021). Discussions could be facilitated by using digital systems prior to the consultation to assess patient readiness and suitability using a standardised approach (Albert et al, 2020).

As in this study, computer-based interventions have previously been proposed to facilitate delivering advice by acting as a prompt (Nápoles et al, 2016). A lack of a consistent contextual cue has been a barrier to discussing PA in different contexts (Dickfos et al, 2015), whereby structured protocols or templates would facilitate delivering brief PA advice (Lamming et al, 2017). Digital systems need to be well-integrated, fit in with and aid the current workflow in order to be effective prompts (Dickfos et al, 2015; Granja et al, 2018). These were also important themes in this study. PA advice also needs to be delivered in the right context to increase patient receptivity (Hall et al, 2021). The ability to adjust the template to suit the consultation and provide a contextual prompt was also a facilitator of using a digital system in this study. Therefore, the use of a digital system as a prompt requires physical opportunity barriers to be addressed. 
The ability to track and share patient data was considered to be a facilitator to using digital systems in this study. Indeed, the inability to monitor follow-up is a barrier for delivering brief PA advice (Hall et al, 2021), which could be overcome by using a digital system to provide a platform to track and monitor patient PA over time at follow-up (Wattanapisit et al, 2021a).

One theme in this study largely unmentioned previously was that digital systems may prevent mistakes and ensure patient safety by addressing the information gaps of HCPs. Additionally, participants highlighted that the time-efficiency of a digital system may depend on the extent of the safety measures used to ensure patient confidentiality.

\section{Implications for research, practice and policy}

The results of this study provides several recommendations for the design of a digital system to support delivering PA advice, by addressing the barriers and leveraging the facilitators. The digital system should be well-integrated into the existing workflow of primary care HCPs, and be quick and easy to use, whilst collecting all relevant data. The digital system should also be flexible to provide a relevant contextual prompt for discussing PA. The digital system should also facilitate conversation between HCPs and patients, by using language that is appropriate and tailored towards the patient. The ability to produce physical print copies of any digital recommendations would assist those HCPs communicating with patients with a lack of motivation to engage with digital systems. Safety features need to be in place for identification and ensuring patient confidentiality, but should not be overly cumbersome. The ease, simplicity and efficiency of digital systems can address some of the barriers facing the delivery of PA advice. However, this requires barriers to using the digital system itself to also be addressed, such as sufficient education and training in digital systems, confidence in using digital systems, or access to in-house support for using the digital system.

As an example, digital systems could be used to generate personalised, printable guides from computer-based assessments of readiness to change and PA levels, as has been recently implemented in the Portuguese NHS (Mendes et al, 2020). The provision of digital templates and elearning within existing platforms could facilitate HCPs lacking in communication skills or knowledge of PA, with a monitoring system to provide follow-up.

Further work should include interdisciplinary collaborations to ensure the digital tool is usable, efficient and that HCPs engage with it to support delivery of PA advice in primary care. Hence, the next step should bring together developers who design digital systems in primary care, the service users (HCPs) of digital systems to consider the user journey and needs, and behavioural scientists to translate design recommendations into tangible prototypes to be tested.

\section{Strengths and limitations}

A strength of this study is the use of a behavioural framework for interviewing and analysis to systematically identify the barriers and facilitators for using digital systems to deliver brief PA advice. The study also asked participants to consider any digital system, where many previous studies have focussed on certain aspects of digital systems, such as eHealth or mHealth interventions.

Limitations include the range of HCP specialisms in this study, which only included GPs, nurses and HCAs, and therefore did not consider the views of other HCPs within primary care. Furthermore, this study was conducted during the initial months of the COVID-19 pandemic, which may have influenced the perceptions of using digital systems. 


\section{Conclusions}

Using a behavioural framework and qualitative approach, this study systematically identified important barriers and facilitators to using digital systems to support delivery of PA advice in primary care. Important themes were found within eight theoretical domains, and most often within physical opportunity. These barriers can be addressed through the design of efficient and flexible digital support tools to facilitate HCPs to deliver PA advice in primary care. To do so, future work needs to combine designers, service users and behavioural scientists to design and develop testable prototypes. 


\section{Funding}

This is part of a wider programme of work (Moving Healthcare Professionals Programme) funded by Sport England.

\section{Conflict of interest}

The authors declare no conflicts of interest.

\section{Ethics statement}

Ethical approval for the study was provided by Public Health England Research Ethics and Governance Group.

Participants provided written informed consent before taking part.

\section{Abbreviations}

GP, general practitioner; HCP, healthcare professional; HCA, healthcare assistant; PA, physical activity 


\section{References}

Michie S, Atkins L, West R. (2014) The Behaviour Change Wheel: A Guide to Designing Interventions. London: Silverback Publishing. www.behaviourchangewheel.com.

Michie, S., van Stralen, M.M. \& West, R. The behaviour change wheel: A new method for characterising and designing behaviour change interventions. Implementation Sci 6, 42 (2011). https://doi.org/10.1186/1748-5908-6-42

Cane, J., O'Connor, D. \& Michie, S. Validation of the theoretical domains framework for use in behaviour change and implementation research. Implementation Sci 7, 37 (2012). https://doi.org/10.1186/1748-5908-7-37

Ryan, G. W., \& Bernard, H. R. (2003). Techniques to Identify Themes. Field Methods, 15(1), 85-109. https://doi.org/10.1177/1525822X02239569

Wattanapisit, A., Thanamee, S., \& Wongsiri, S. (2019). Physical activity counselling among GPs: a qualitative study from Thailand. BMC family practice, 20(1), 72. https://doi.org/10.1186/s12875-019-0968-x

Huntington, J., Dwyer, J.J.M., Shama, S. et al. Registered dietitians' beliefs and behaviours related to counselling patients on physical activity and sedentary behaviour from a theory of planned behaviour perspective. BMC Nutr 6, 66 (2 020). https://doi.org/10.1186/s40795-020$\underline{00392-1}$

Gustavsson, C., Nordqvist, M., Bröms, K. et al. What is required to facilitate implementation of Swedish physical activity on prescription? - interview study with primary healthcare staff and management. BMC Health Serv Res 18, 196 (2018). https://doi.org/10.1186/s12913-018$\underline{3021-1}$

Jones, M., Bright, P., Hansen, L., Ihnatsenka, O., \& Carek, P. J. (2021). Promoting Physical Activity in a Primary Care Practice: Overcoming the Barriers. American Journal of Lifestyle Medicine, 15(2), 158-164. https://doi.org/10.1177/1559827619867693

Milton, K., Cavill, N., Chalkley, A., Foster, C., Gomersall, S., Hagstromer, M., Kelly, P., KolbeAlexander, T., Mair, J., McLaughlin, M., Nobles, J., Reece, L., Shilton, T., Smith, B. J., \& Schipperijn, J. (2021). Eight Investments That Work for Physical Activity, Journal of Physical Activity and Health, 18(6), 625-630. Retrieved Sep 8, 2021, from https://journals.humankinetics.com/view/journals/jpah/18/6/article-p625.xml

Loprinzi PD, Beets MW. Need for increased promotion of physical activity by health care professionals. Prev Med. 2014 Dec;69:75-9. doi: 10.1016/j.ypmed.2014.09.002. Epub 2014 Sep 16. PMID: 25230367.

Albert, F.A.; Crowe, M.J.; Malau-Aduli, A.E.O.; Malau-Aduli, B.S. Physical Activity Promotion: A Systematic Review of The Perceptions of Healthcare Professionals. Int. J. Environ. Res. Public Health 2020, 17, 4358. https://doi.org/10.3390/ijerph17124358

Hall, L. H., Thorneloe, R., Rodriguez-Lopez, R., Grice, A., Thorat, M. A., Bradbury, K., Wadnerkar Kamble, M., Okoli, G. N., Powell, D., \& Beeken, R. J. (Accepted/ln press). Delivering brief physical activity interventions in primary care: a systematic review of the prevalence, and factors associated with delivery, receipt, and patient receptivity. The British Journal of General Practice. 
NICE. Physical activity: brief advice for adults in primary care. Public health guideline [PH44] 1 Recommendations | Physical activity: brief advice for adults in primary care | Guidance NICE

Dickfos M, King D, Parekh S, Boyle FM, Vandelanotte C. General practitioners' perceptions of and involvement in health behaviour change: can computer-tailored interventions help? Prim Health Care Res Dev. 2015 May;16(3):316-21. doi: 10.1017/S1463423614000498. Epub 2014 Nov 13. PMID: 25391284.

Christian JG, Byers TE, Christian KK, Goldstein MG, Bock BC, Prioreschi B, Bessesen DH. A computer support program that helps clinicians provide patients with metabolic syndrome tailored counseling to promote weight loss. J Am Diet Assoc. 2011 Jan;111(1):75-83. doi: 10.1016/j.jada.2010.10.006. PMID: 21185968.

2020a Wattanapisit, A., Tuangratananon, T., \& Wattanapisit, S. (2020a). Usability and utility of eHealth for physical activity counselling in primary health care: a scoping review. BMC family practice, 21(1), 229. https://doi.org/10.1186/s12875-020-01304-9

2020b Wattanapisit A, Teo CH, Wattanapisit S, Teoh E, Woo WJ, Ng CJ. Can mobile health apps replace GPs? A scoping review of comparisons between mobile apps and GP tasks. BMC Med Inform Decis Mak. 2020b Jan 6;20(1):5. doi: 10.1186/s12911-019-1016-4. PMID: 31906985; PMCID: PMC6945711.

2021a Wattanapisit, A., Wattanapisit, S., Tuangratananon, T., Amaek, W., Wongsiri, S., \& Petchuay, P. (2021a). Primary Health Care Providers' Perspectives on Developing an eHealth Tool for Physical Activity Counselling: A Qualitative Study. Journal of multidisciplinary healthcare, 14, 321-333. https://doi.org/10.2147/JMDH.S298390

2021b Wattanapisit, A., Amaek, W., Wattanapisit, S., Tuangratananon, T., Wongsiri, S., \& Pengkaew, P. (2021b). Challenges of Implementing an mHealth Application for Personalized Physical Activity Counselling in Primary Health Care: A Qualitative Study. International journal of general medicine, 14, 3821-3831. https://doi.org/10.2147/IJGM.S317241

2021c Wattanapisit, A., Wattanapisit, S., \& Wongsiri, S. (2021c). Overview of Physical Activity Counseling in Primary Care. Korean journal of family medicine, 42(4), 260-268. https://doi.org/10.4082/kjfm.19.0113

Goryakin Y, Suhlrie L, Cecchini M. Impact of primary care-initiated interventions promoting physical activity on body mass index: systematic review and meta-analysis. Obes Rev. 2018 Apr;19(4):518-528. doi: 10.1111/obr.12654. Epub 2018 Jan 23. PMID: 29363253.

Lamming L, Pears S, Mason D, Morton K, Bijker M, Sutton S, Hardeman W; VBI Programme Team. What do we know about brief interventions for physical activity that could be delivered in primary care consultations? A systematic review of reviews. Prev Med. 2017 Jun;99:152163. doi: 10.1016/j.ypmed.2017.02.017. Epub 2017 Feb 21. PMID: 28232098.

Law R, Langley J, Hall B, et al. 'Function First': how to promote physical activity and physical function in people with long-term conditions managed in primary care? A study combining realist and co-design methods. BMJ Open 2021;11:e046751. doi: 10.1136/bmjopen-2020046751

Mendes, R.; Nunes Silva, M.; Santos Silva, C.; Marques, A.; Godinho, C.; Tomás, R.; Agostinho, M.; Madeira, S.; Rebelo-Marques, A.; Martins, H.; Teixeira, P.J.; Cruz, D. Physical Activity Promotion Tools in the Portuguese Primary Health Care: An Implementation 
Research. Int. J. Environ. Res. Public Health 2020, 17, 815. https://doi.org/10.3390/ijerph17030815

Netherway, J.; Smith, B.; Monforte, J. Training Healthcare Professionals on How to Promote Physical Activity in the UK: A Scoping Review of Current Trends and Future Opportunities. Int. J. Environ. Res. Public Health 2021, 18, 6701. https://doi.org/10.3390/ijerph18136701

Vishnubala D, Pringle A. Working with healthcare professionals to promote physical activity. Perspect Public Health. 2021 Mar;141(2):111-113. doi: 10.1177/1757913920978253. Epub 2021 Feb 11. PMID: 33573514.

O'Regan, A., Pollock, M., D'Sa, S., \& Niranjan, V. (2021). ABC of prescribing exercise as medicine: a narrative review of the experiences of general practitioners and patients. $B M J$ open sport \& exercise medicine, 7(2), e001050. https://doi.org/10.1136/bmisem-2021-001050

Nápoles, A. M., Appelle, N., Kalkhoran, S., Vijayaraghavan, M., Alvarado, N., \& Satterfield, J. (2016). Perceptions of clinicians and staff about the use of digital technology in primary care: qualitative interviews prior to implementation of a computer-facilitated $5 \mathrm{As}$ intervention. $B M C$ medical informatics and decision making, 16, 44. https://doi.org/10.1186/s12911-016-0284-5

Poppe, L., Plaete, J., Huys, N., Verloigne, M., Deveugele, M., De Bourdeaudhuij, I., \& Crombez, G. (2018). Process Evaluation of an eHealth Intervention Implemented into General Practice: General Practitioners' and Patients' Views. International journal of environmental research and public health, 15(7), 1475. https://doi.org/10.3390/ijerph15071475

Granja, C., Janssen, W., \& Johansen, M. A. (2018). Factors Determining the Success and Failure of eHealth Interventions: Systematic Review of the Literature. Journal of medical Internet research, 20(5), e10235. https://doi.org/10.2196/10235

Brannan, M., Bernardotto, M., Clarke, N. et al. Moving healthcare professionals - a whole system approach to embed physical activity in clinical practice. BMC Med Educ 19, 84 (2019). https://doi.org/10.1186/s12909-019-1517-y

Degroote, L., Van Dyck, D., De Bourdeaudhuij, I., De Paepe, A., \& Crombez, G. (2020). Acceptability and feasibility of the mHealth intervention 'MyDayPlan' to increase physical activity in a general adult population. BMC public health, 20(1), 1032. https://doi.org/10.1186/s12889-020-09148-9

Global action plan on physical activity 2018-2030: more active people for a healthier world. Geneva: World Health Organization; 2018. Licence: CC BY-NC-SA 3.0 IGO. Available from: https://apps.who.int/iris/bitstream/handle/10665/272722/9789241514187-eng.pdf (accessed September 9th, 2021)

Boudreau, F., Dagenais, G. R., de Vries, H., Walthouwer, M., Côté, J., Turbide, G., Bourlaud, A. S., \& Poirier, P. (2020). Effectiveness of a web-based computer-tailored intervention promoting physical activity for adults from Quebec City: a randomized controlled trial. Health psychology and behavioral medicine, 8(1), 601-622. https://doi.org/10.1080/21642850.2020.1850287

Verwey, R., van der Weegen, S., Spreeuwenberg, M., Tange, H., van der Weijden, T., \& de Witte, L. (2016). Process evaluation of physical activity counselling with and without the use of mobile technology: A mixed methods study. International journal of nursing studies, 53, 316. https://doi.org/10.1016/.ijnurstu.2015.10.008 
Neudorf, B., Giangregorio, L., \& Morita, P. (2020). Insights From an Usability Review of an Electronic Medical Record-Integrated Physical Activity Counseling Tool for Primary Care. Ergonomics in Design: The Quarterly of Human Factors Applications. 106480462098214. 10.1177/1064804620982140.

van der Weegen S, Verwey R, Spreeuwenberg M, Tange $H$, van der Weijden $T$, de Witte L. It's LiFe! Mobile and Web-Based Monitoring and Feedback Tool Embedded in Primary Care Increases Physical Activity: A Cluster Randomized Controlled Trial. J Med Internet Res. 2015 Jul 24;17(7):e184. doi: 10.2196/jmir.4579. PMID: 26209025; PMCID: PMC4529491.

World Health Organisation (WHO). Global health risks: mortality and burden of disease attributable to selected major risks. 2009. Available from: https://www.who.int/healthinfo/global burden disease/GlobalHealthRisks report full.pdf?ua (accessed September $6^{\text {th }}, 2021$ )

Wahid A, Manek N, Nichols M, Kelly P, Foster C, Webster P, et al. Quantifying the Association Between Physical Activity and Cardiovascular Disease and Diabetes: A Systematic Review and Meta-Analysis. J Am Heart Assoc 2016;5. https://doi.org/10.1161/JAHA.115.002495.

GOV.UK. Physical activity guidelines: UK Chief Medical Officers' report. Available from: https://www.gov.uk/government/publications/physical-activity-guidelines-uk-chief-medicalofficers-report (accessed February 8, 2020).

2018 Physical Activity Guidelines Advisory Committee. 2018 Physical Activity Guidelines Advisory Committee Scientific Report. Washington, DC: U.S. Department of Health and Human Services, 2018.

F. Campbell, L. Blank, J. Messina, M. Day, H.B. Wood, N. Payne, E. Goyder, C. Armitage. 2012. Physical Activity: Brief Advice for Adults in Primary Care (National Institute for Health and Clinical Excellence Public Health Intervention Guidance. https://www.nice.org.uk/guidance/ph44/evidence/review-of-effectiveness-and-barriers-andfacilitators-69102685

2019a Thorneloe, R.J., Lamb, M., Stevens, R., Harrop, D., \& Arden, M. (2019a) Tools and solutions developed to support the delivery of brief physical activity interventions by healthcare professionals: An Evidence Report. Public Health England.

2019b Thorneloe, R.J., Lamb, M., Stevens, R., Harrop, D., \& Arden, M. (2019b) Exploring the barriers and facilitators to delivering brief physical activity interventions in primary care: Rapid review and primary insight study. Public Health England.

Atkins, L., Francis, J., Islam, R. et al. A guide to using the Theoretical Domains Framework of behaviour change to investigate implementation problems. Implementation Sci 12, 77 (2017). https://doi.org/10.1186/s13012-017-0605-9

Rosário, F., Santos, M.I., Angus, K. et al. Factors influencing the implementation of screening and brief interventions for alcohol use in primary care practices: a systematic review using the COM-B system and Theoretical Domains Framework. Implementation Sci 16, 6 (2021). https://doi.org/10.1186/s13012-020-01073-0

Hall, A.M., Scurrey, S.R., Pike, A.E. et al. Physician-reported barriers to using evidence-based recommendations for low back pain in clinical practice: a systematic review and synthesis of qualitative studies using the Theoretical Domains Framework. Implementation Sci 14, 49 (2019). https://doi.org/10.1186/s13012-019-0884-4 
Agarwal P, Kithulegoda N, Bouck Z, Bosiak B, Birnbaum I, Reddeman L, Steiner L, Altman L, Mawson R, Propp R, Thornton J, Ivers N. Feasibility of an Electronic Health Tool to Promote Physical Activity in Primary Care: Pilot Cluster Randomized Controlled Trial. J Med Internet Res 2020;22(2):e15424

GC Vijay Wilson EC, Suhrcke M, Hardeman W, Sutton S; VBI Programme Team. Are brief interventions to increase physical activity cost-effective? A systematic review. Br J Sports Med. 2016 Apr;50(7):408-17. doi: 10.1136/bjsports-2015-094655. Epub 2015 Oct 5. PMID: 26438429; PMCID: PMC4819643. 